\title{
THE IDEA OF A TECHNOLOGY FOR EDUCATION AND TRAINING
}

\author{
RichaRd GoOdMan, M.A., B.Sc. \\ Department of Computing, Cybernetics and \\ Management, Brighton College of Technology
}

THE idea that we should now consciously set about creating an adequate modern technology for education has largely grown up since the last war. But it is only over the past few years that there has been an appreciable quickening of interest and of effort. This forward move has not been based on grounds of expediency alone-the exponential growth of knowledge, the persistent and growing teacher shortage, and the extreme inefficiency of current methods. The development of the new sciences of communication and control, research into self-adapting and self-organising systems, our growing "know how" about the mass media, exploration of the potential of sophisticated computing and data processing systems and the elaboration of simulation techniques, all have immense implications and make possible radical, qualitative changes.

Of course there has always been a technology of education. But it has been, and is still predominantly, a tool-technology, a technology of "audio-visual aids". My thesis here is that such a tool-technology is not enough. What is required today and especially tomorrow is a systems-technology, a technology based not only upon support-systems but itself also consciously applying the systems-concept.

Here it is necessary to make clear the sense in which we are using the terms "tool", "system" and "technology".

Unaided, a man, even if he is a Valerie Brumel, cannot jump more than about seven feet from the earth's surface. Using a fibre glass pole, however, even quite ordinary athletes can clear double this height. But if we want to move farther away from the earth, we have to use helicopters, airplanes and rockets. The fibre-glass pole is a tool; the helicopter with pilot, the rocket, with or without cosmonaut, is a system and not only a system in itself but a system functioning effectively only within a nested set of wider systems. In attempting to create a modern technology for education, we have to differentiate between the tools, the audio-visual aids as we have called them, and the support systems now becoming available.

Again, by a technology, we do not mean just a collection of hardware. It is customary to think of technology as somewhat dehuman, even antihuman. This is a monstrous error. Technology, I suggest, is that complex of human activities concerned to use the findings of science to solve the problems of human society. The technologist is essentially a $\overline{0}$ humanist. A technology is, therefore, a social activity, involving people and ideas, methods and machines, dynamic structures, communications, values and tools; but always people. Its objective is to achieve an efficient and rational division of labour, subject to human control, $\infty$ between people and the support systems they 은 evolve, to amplify human creativity, not to replace it.

Concerned then with the elaboration of support systems to achieve essentially human ends, $\overrightarrow{0}$ such a technology must emphasize the absolute or need both to state objectives in detailed, ade quately operational terms and to test anf validate the means developed to achieve thent. This is one of the outstanding characteristics of the new thinking: that the science of communication and control is applicable to the teaching-learning process just as to any other adaptive process.

It is against such a background of ideas that we must assess the growing range of support systems now becoming available to the human teacher. And it is this approach that will check any tendency to advocate one particular brand of device occupying one band only of the entire spectrum of resources as a gimmick panacea for all our educational ills. Some over-

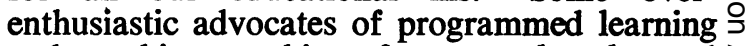
and teaching machines for example, obsessed $\frac{7}{0}$ by the notion of closed-loop systems (though none of the general-purpose teaching machines $N$ in existence today is really an example of such a system), have tended, at least by implication, $N$ to dismiss open-ended systems, like television $\omega$ and the lecture, as educationally of little value. But this is a dangerous and demonstrable fallacy: even systems open-ended in themselves will, when embedded into wider systems, also provide feedback, control information. And in $\frac{0}{\circ}$ the very complex process, teaching-learning, a wide range of sub-processes is involved, not all 
of which, in themselves, require closed-loop working.

Clearly, if we are to develop an adequate, modern technology for education we have to be pretty clear about the function of the human educationalist, the human teacher. It is a pity that so much discussion about educational technology and especially about that band of our systems-spectrum occupied by programmed learning and teaching machines has been so closely linked with the ideas of a particular school of animal psychologists. The trouble is, as Wall and Meredith have pointed out, that rats and pigeons are not social creatures, while human learning is essentially a social process. This is not to deny the important role played by conditioning in human learning but conditioning is not knowledge, it is one of the foundations upon which knowledge is grown. It is probable, indeed, that individual psychology (and most animal psychology is individual psychology) will never be able to develop an adequate learning theory: as individual psychology it is bound to underweight the social factors.

We can put all this slightly differently: It may be possible to acquire an appropriate verbal behaviour, a "knowledge" of concepts and procedures in, say, an individual tutorial situation, but this knowledge is only consolidated and real learning only takes place when these concepts, skills and procedures are applied to problem-solving and decision-making situations within the context of a social group or team. And this is true, I suggest, even when the subjects are highly verbal, like philosophy. In other words, we have, I think, to recognise that the core or nucleus of any true learning situation is the activity of a social group of interacting people, with the human teacher operating to some degree or another as the essential and irreplaceable catalyst. The individual one-tutor-one student situation, "the classical process of individual tutoring", in Crowder's words, is not the crowning learning situation. But having said this, we must hasten to add that, in so doing, we are not denying its diagnostic, therapeutic, remedial function. Nor, since different people acquire knowledge of concepts, skills, procedures, of the syntax and semantics of a subject, at different speeds, are we denying that it plays a key role in this phase of the overall learning process. What we are saying, among other things, is that programmed instruction and teaching machines, at their present level of development certainly, are neither so self-sufficient nor as self-contained as has been claimed. (In this connection it is interesting to note that Systems Development Corporation has recently been given a grant by the U.S. Office of Education to explore the effects of teacher intervention to augment programmed instruction, the extent to which the teacher's effectiveness increases by providing current information about individual student progress, and the effects of allowing students partial control over programme sequence).

May I try to put my point in another way? The function of the human teacher (and we should stress here that the design of a course, the specification of its objectives and, to a large extent, its validation will always be a human responsibility) is to create a set of simplified but necessarily dynamic environments, models, situations, through which the learners are guided, as minimally as possible, to explore. The actual exploration is a social process; the antecedent training and briefing, the individual preparation, is where our new communication and control systems can contribute, almost without limit. But even here we have to remember that even when physically alone the student is still a social being.

Such preparatory training and briefing, such acquisition of concepts, procedures and skill can be divided roughly into three phases (necessarily overlapping and interacting):

that involving the acquisition of back? ground knowledge and perspectives, the development of a climate of relevance to the real world, and the build up of what may be called contextual literacy (where, of course, motivation is a key question); that involving the acquisition of concepts, skills, procedures (what we have called the "syntax and semantics" of the appropriate "language" or "languages"); and that constituting the "tryout" of all this in practice, its application to a problemsolving, decision-making situation that is "real" to the learners, a "play" situation, if you like.

For the first phase, as we have called it, the open-ended systems (television, the film, the lecture in its "inspirational" function, background reading of conventional books, etc.) are specially suited. The need for immediate control feedback is not here so extreme. The details of the distant prospects of a landscape are not as important as the general excitement created. But if the entire project is conceived and designed systems-wise, cybernetically, an adequate, longer-termed, feedback can be obtained and will be sufficient for evaluation and subsequent adaptation. 
For the second phase, where individual factors enter more decisively into the situation, especially speed of learning, previous knowledge, etc., more immediate and detailed feedback is required. Here is where programmed instruction and teaching machines can contribute immediately and effectively. And this is so especially in those sectors at which the human teacher does not excel (e.g. information-imparting) and with which, because of the exigencies of the situation, we cannot be expected adequately to cope. But it will contribute effectively if, and only if, at this level as well, an adequate systems-analysis is carried out. For instance, we have to examine very carefully exactly what job we want each programme sequence to perform, by what means they will be "called", and we have to ask ourselves to what extent each sequence shall be "extraverted", if I may use that term, or refer outwards from itself to other procedures, like reading, lectures, seminars, and laboratory experiments. In short, it is necessary to regard our programmed sequences something like "subroutines" within a wider, perhaps very complicated and versatile programme. Moreover, it is at this stage that we have to ask ourselves which such "subroutines" are available commercially and which have to be specially written

For the third phase, the phase of "try-out" and "play", preceding actual exploration, simulation techniques and simulation systems are required I think that inadequate attention has been paid to this phase and to the potentialities of these techniques and systems. Apart from the opportunity it provides for acquiring the necessary skills in handling "apparatus", laboratory work, for example, falls essentially in this phase. It is consequently quite futile if it consists solely in the repetition ad nauseam of "problems" or "experiments" which have been performed time and time again. But those who, for this reason, advocate a reduction in "practical work", in "lab. time", forget that it is possible, and, indeed, essential, to set problems that are "real" to the student at his current level of development and, consequently, may be very difficult of "solution". Moreover such problems should be set to groups of students rather than to individuals. A group assigned the problem of finding the eigenvalues of a matrix, for instance, will learn more if, instead of being "cooked" to give easily isolable eigen-values, the given matrix is such that, for example, two of the values lie very close together and the group's repertoire of techniques is stretched to its limits in the attempt to isolate them.
Simulation techniques and systems are especially important in those "subjects" where the opportunity to tackle "real problems" is restricted or, perhaps, impracticable (e.g. in the social sciences, management studies and, I would suggest, certain fields of medicine). Here, too, the computer is an excellent and versatile supporting device. Computerised simulations requiring active student participation in groups and a considerable degree of role-playing are very powerful allies of the human teacher. Indeed, they may be used, as we are trying to use them at Brighton, as the integrating system for an entire course, coordinating the use of a whole range of subsystems, like closed circuit television, teaching machines, seminars, tutorials and, even, "private study". Unfortunately I have no time to discuss these methods in detail, but $I$ do urge their serious investigation.

Of course these three phases are not serially organised, they are intermingled and interactive, and, as such, pose very considerable control problems. Here, too, it appears the human teacher will require to call upon the computer for support and assistance. But we are now, you see, beginning to be confronted with some of the wider implications of the development of a modern technology for educa tion. We cannot here attempt even to stare considering these implications in depth. I shall conclude. however, by briefly mentioning just three, picked more or less at random.

First, there is the problem of retraining our teachers at all levels, including those at universities and other higher institutions, to think in terms of a system technology for education and to be "literate" in this field. This cannot be solved by ad hoc casual and local measures; it is essentially a national problem.

Secondly, there is the problem of providing an adequate number of technicians to maintain, operate and develop these new systems. This cannot be left to the teacher or solved by the appointment as local "audio-visual aids officers" of ex-sergeants-major!

Finally, there is the problem of the functional design of new institutes of learning. Perhaps I am unduly pessimistic, but it appears to me that, for instance, not one of our "new" universities will escape educational and architectural obsolescence in five years from now.

The idea of a modern technology for education, if not revolutionary in itself, has at least profoundly revolutionary implications. 